\title{
Statistical Analysis of Ratio of Random Variables and Its Application in Performance Analysis of Multihop Wireless Transmissions
}

\author{
Edis Mekić, ${ }^{1}$ Mihajlo Stefanović, ${ }^{2}$ Petar Spalević, ${ }^{3}$ \\ Nikola Sekulović, ${ }^{2}$ and Ana Stanković ${ }^{2}$ \\ ${ }^{1}$ Department of Computer Technologies, State University of Novi Pazar, Vuka Karadzica bb, \\ 36300 Novi Pazar, Serbia \\ ${ }^{2}$ Faculty of Electronic Engineering, University of Nis, Aleksandra Medvedeva 14, 18000 Nis, Serbia \\ ${ }^{3}$ Faculty of Technical Sciences, University of Kosovska Mitrovica, Kneza Milosa 7, \\ 38220 Kosovska Mitrovica, Serbia
}

Correspondence should be addressed to Edis Mekić, emekic@np.ac.rs

Received 2 July 2011; Accepted 19 October 2011

Academic Editor: Kue-Hong Chen

Copyright (C) 2012 Edis Mekić et al. This is an open access article distributed under the Creative Commons Attribution License, which permits unrestricted use, distribution, and reproduction in any medium, provided the original work is properly cited.

The distributions of random variables are of interest in many areas of science. In this paper, the probability density function (PDF) and cumulative distribution function (CDF) of ratio of products of two random variables and random variable are derived. Random variables are described with Rayleigh, Nakagami- $m$, Weibull, and $\alpha-\mu$ distributions. An application of obtained results in performance analysis of multihop wireless communication systems in different transmission environments described in detail . The proposed mathematical analysis is also complemented by various graphically presented numerical results.

\section{Introduction}

The distribution of the ratio of random variables is of interest in statistical analysis in biological and physical sciences, econometrics, and ranking and selection [1]. It has been studied by several authors [2-5]. The ratio of random variables is also of interest in analysing wireless communication systems [6-9]. In wireless communication systems, one of problems is fading caused by multipath propagation. When a received signal experiences fading during transmission, signal envelope fluctuates over time [10]. There is a very wide range of statistical models for describing the statistical behaviour of signal envelope where accuracy and veracity depend on communication scenario and propagation environment. The most frequently applied models in the open technical literature are Rayleigh, Nakagami$m$, Weibull, and $\alpha-\mu$. In addition to fading, the wireless transmission can be also subjected 
to shadowing which is the result of large obstacles and deviations in terrain profile between transmitter and receiver. In such composite environments, signal envelope can be modelled with product of two random variables [11, 12]. A fundamental requirement in planning wireless communication systems, besides providing specified quality of service (QoS), is to ensure high system capacity. Cochannel interference (CCI) is a result of frequency reuse which is essential in increasing system capacity [13].

In the areas of deployment, connectivity, and capacity, multihop systems have a number of advantages over traditional communication networks. Relaying techniques enable network connectivity where traditional architectures are impractical due to location constraints and can be applied to cellular, wireless local area networks (WLANs) and hybrid networks. The performance analysis of multihop wireless communication systems operating in fading channels has been an important field of research in the past few years [14-17].

The main contribution of this paper is the derivation of useful closed-form expressions for the probability density function (PDF) and cumulative distribution function (CDF) of ratio of product of two random variables and random variable. Among the rest, these expressions can be used as general theoretical tool in the performance analysis of wireless communication systems since the product of random variables in nominator can represent signal envelope which suffers from fading and shadowing while the random variable in denominator can represent CCI envelope. Therefore, the ratio of such random variables is signal-to-interference ratio (SIR). Rayleigh, Nakagami- $m$, Weibull, and $\alpha-\mu$ distributions are included in our analysis to enable wide range of use of results presented in the paper, that is, to model different transmission environments. Capitalizing on closed-form expressions derived in the paper in the terms of Meijer G functions, the outage probability of multihop system, as an important and widely accepted performance measure, is determined and discussed.

\section{Statistics of Ratio of Products of Two Random Variables and Random Variable}

In this section, statistical analysis of ratio of products of two random variables and random variable, $\lambda=x z / y$, is presented. For derivation of the corresponding PDF of $\lambda$, we have to obtain the PDF of products of two random variables, $t=x z$, using the following equation:

$$
p_{t}(t)=\int_{0}^{\infty}|J| p_{x}\left(\frac{t}{z}\right) p_{z}(z) d z
$$

where $|J|$ is the Jacobian transformation given by $|J|=|d x / d t|=1 / z$. Using similar procedure, the PDF of $\lambda$ can be derived as

$$
p_{\lambda}(\lambda)=\int_{0}^{\infty}|J| p_{t}(\lambda y) p_{y}(y) d y,
$$

where $|J|=|d t / d \lambda|=y$. 
Cumulative distribution function can be obtained by definition as

$$
F_{\lambda}(\lambda)=\int_{0}^{\lambda} p_{\lambda}(\lambda) d \lambda
$$

First, we present different distributions describing statistical behaviour of variables $x, y$, and $z$.

\section{(a) Rayleigh Distribution}

In the case of Rayleigh distribution, the PDFs of random variables are given by

$$
\begin{aligned}
& p_{x}(x)=\frac{2}{\Omega_{x}} x e^{-x^{2} / \Omega_{x}}, \\
& p_{y}(y)=\frac{2}{\Omega_{y}} y e^{-y^{2} / \Omega_{y}}, \\
& p_{z}(z)=\frac{2}{\Omega_{z}} z e^{-z^{2} / \Omega_{z}},
\end{aligned}
$$

where $\Omega_{x}=\varepsilon\left\langle x^{2}\right\rangle, \Omega_{y}=\varepsilon\left\langle y^{2}\right\rangle, \Omega_{z}=\varepsilon\left\langle z^{2}\right\rangle$, and $\varepsilon\langle\cdot\rangle$ denotes expectation.

(b) Weibull Distribution

The Weibull random variables are distributed according to

$$
\begin{aligned}
& p_{x}(x)=\frac{\beta}{\Omega_{x}} x^{\beta-1} e^{-x^{\beta} / \Omega_{x}}, \\
& p_{y}(y)=\frac{\beta}{\Omega_{y}} y^{\beta-1} e^{-y^{\beta} / \Omega_{y}}, \\
& p_{z}(z)=\frac{\beta}{\Omega_{z}} z^{\beta-1} e^{-z^{\beta} / \Omega_{z}},
\end{aligned}
$$

where $\Omega_{x}=\varepsilon\left\langle x^{\beta}\right\rangle, \Omega_{y}=\varepsilon\left\langle y^{\beta}\right\rangle, \Omega_{z}=\varepsilon\left\langle z^{\beta}\right\rangle$, and $\beta$ is Weibull parameter (for the special case of $\beta=2$ Weibull PDFs reduces to Rayleigh PDFs).

(c) Nakagami-m Distribution

The Nakagami- $m$ PDFs are

$$
\begin{aligned}
& p_{x}(x)=\frac{2 m_{x}^{m_{x}}}{\Gamma\left(m_{x}\right) \Omega_{x}^{m_{x}}} x^{2 m_{x}-1} e^{-m_{x} x^{2} / \Omega_{x}}, \\
& p_{y}(y)=\frac{2 m_{y}^{m_{y}}}{\Gamma\left(m_{y}\right) \Omega_{y}^{m_{y}}} y^{2 m_{y}-1} e^{-m_{y} y^{2} / \Omega_{y}}, \\
& p_{z}(z)=\frac{2 m_{z} m_{z}}{\Gamma\left(m_{z}\right) \Omega_{z}^{m_{z}}} z^{2 m_{z}-1} e^{-m_{z} z^{2} / \Omega_{z}},
\end{aligned}
$$


where $\Omega_{x}=\varepsilon\left\langle x^{2}\right\rangle, \Omega_{y}=\varepsilon\left\langle y^{2}\right\rangle, \Omega_{z}=\varepsilon\left\langle z^{2}\right\rangle, \Gamma(\cdot)$ is gamma function, and $m_{x}, m_{y}$, and $m_{z}$ are Nakagami- $m$ parameters that range from 0.5 to $\infty$. Nakagami- $m$ distribution spans via $\mathrm{m}$ parameter through wide range of distributions which includes the one-sided Gaussian distribution $(m=0.5)$ and the Rayleigh distribution $(m=1)$ as special cases.

\section{(d) $\alpha-\mu$ Distribution}

The $\alpha-\mu$ distributed random variables are described with following equations:

$$
\begin{aligned}
& p_{x}(x)=\alpha\left(\frac{\mu_{x}}{\Omega_{x}}\right)^{\mu_{x}} \frac{x^{\alpha \mu_{x}-1}}{\Gamma\left(\mu_{x}\right)} e^{-\mu_{x} x^{\alpha} / \Omega_{x}}, \\
& p_{y}(y)=\alpha\left(\frac{\mu_{y}}{\Omega_{y}}\right)^{\mu_{y}} \frac{y^{\alpha \mu_{y}-1}}{\Gamma\left(\mu_{y}\right)} e^{-\mu_{y} y^{\alpha} / \Omega_{y}}, \\
& p_{z}(z)=\alpha\left(\frac{\mu_{z}}{\Omega_{z}}\right)^{\mu_{z}} \frac{z^{\alpha \mu_{z}-1}}{\Gamma\left(\mu_{z}\right)} e^{-\mu_{z} z^{\alpha} / \Omega_{z}},
\end{aligned}
$$

where $\Omega_{x}=\varepsilon\left\langle x^{\alpha}\right\rangle, \Omega_{y}=\varepsilon\left\langle y^{\alpha}\right\rangle, \Omega_{z}=\varepsilon\left\langle z^{\alpha}\right\rangle, \alpha$ is parameter related to the nonlinearity, and $\mu_{x}, \mu_{y}$, and $\mu_{z}$ are the inverse of the normalized variance of $x^{\alpha}, y^{\alpha}$ and $z^{\alpha}$, respectively $\left(\mu_{x}, \mu_{y}, \mu_{z} \geq 0.5\right)$. The $\alpha-\mu$ distribution is a general distribution that includes as special cases Nakagami- $m$ distribution for $\alpha=2$ and Weibull distribution for $\mu=1$.

Applying the procedure described at the beginning of this section with the aid of [18, Equations (3.461) and (6.631)], [19], [20, Equation (26)], the PDFs and CDFs of $\lambda$ in different scenarios can be expressed in terms of Meijer $G$ functions $G_{p, q}^{m, n}\left(\gamma \mid \begin{array}{l}a_{1}, a_{2}, \ldots, a_{p} \\ b_{1}, b_{2}, \ldots, b_{q}\end{array}\right)$ as

(a) Rayleigh Scenario

$$
\begin{aligned}
& p_{\lambda}(\lambda)=2 \sqrt{\frac{\Omega_{y}}{\Omega_{x} \Omega_{z}}} G_{1,2}^{2,1}\left(\frac{\Omega_{y}}{\Omega_{x} \Omega_{z}} \lambda^{2} \mid \begin{array}{c}
-\frac{1}{2} \\
\frac{1}{2}, \frac{1}{2}
\end{array}\right),
\end{aligned}
$$

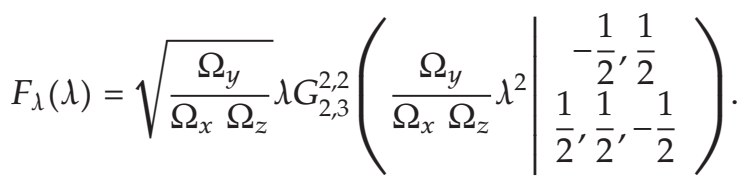

(b) Weibull Scenario

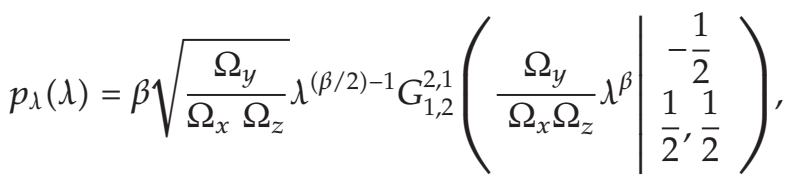

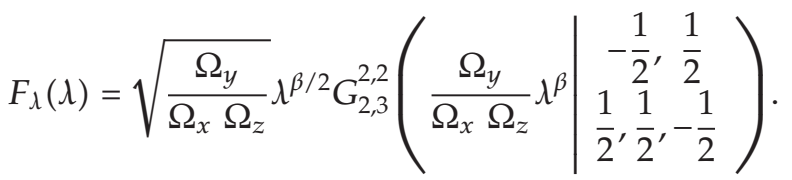


(c) Nakagami-m Scenario

$$
\begin{aligned}
p_{\lambda}(\lambda)= & 2\left(\frac{m_{x} m_{z}}{m_{y}}\right)^{(1 / 2)\left(m_{x}+m_{z}-1\right)}\left(\frac{\Omega_{y}}{\Omega_{x} \Omega_{z}}\right)^{(1 / 2)\left(m_{x}+m_{z}-1\right)} \frac{1}{\Gamma\left(m_{x}\right) \Gamma\left(m_{y}\right) \Gamma\left(m_{z}\right)} \lambda^{m_{x}+m_{z}-2} \\
& \times G_{1,2}^{2,1}\left(\frac{m_{x} m_{z}}{m_{y}} \frac{\Omega_{y}}{\Omega_{x} \Omega_{z}} \lambda^{2} \mid \begin{array}{c}
-\frac{1}{2}\left(m_{x}+2 m_{y}+m_{z}-3\right) \\
\frac{1}{2}\left(m_{z-} m_{x}+1\right), \frac{1}{2}\left(1-m_{z+} m_{x}\right)
\end{array}\right), \\
F_{\lambda}(\lambda)= & \left(\frac{m_{x} m_{z}}{m_{y}}\right)^{(1 / 2)\left(m_{x}+m_{z}-1\right)}\left(\frac{\Omega_{y}}{\Omega_{x} \Omega_{z}}\right)^{(1 / 2)\left(m_{x}+m_{z}-1\right)} \lambda^{m_{x_{j}}+m_{z j}-1} \frac{1}{\Gamma\left(m_{x}\right) \Gamma\left(m_{y}\right) \Gamma\left(m_{z}\right)} \\
& \times G_{2,3}^{2,2}\left(\frac{m_{x} m_{z}}{m_{y}} \frac{\Omega_{y}}{\Omega_{x} \Omega_{z}} \lambda^{2} \mid \begin{array}{l}
-\frac{1}{2}\left(m_{x}+2 m_{y}+m_{z}-3\right), \frac{1}{2}\left(3-m_{x}-m_{z}\right) \\
\frac{1}{2}\left(m_{z}-m_{x}+1\right), \frac{1}{2}\left(1-m_{z}+m_{x}\right),-\frac{1}{2}\left(m_{x}+m_{z}-1\right)
\end{array}\right) .
\end{aligned}
$$

(d) $\alpha-\mu$ Scenario

$$
\begin{aligned}
p_{\lambda}(\lambda)= & \alpha\left(\frac{\mu_{x} \mu_{z}}{\mu_{y}}\right)^{(1 / 2)\left(\mu_{x}+\mu_{z}-1\right)}\left(\frac{\Omega_{y}}{\Omega_{x} \Omega_{z}}\right)^{(1 / 2)\left(\mu_{x}+\mu_{z}-1\right)} \lambda^{(\alpha / 2)\left(\mu_{x}+\mu_{z}-1\right)-1} \\
& \times \frac{1}{\Gamma\left(\mu_{x}\right) \Gamma\left(\mu_{y}\right) \Gamma\left(\mu_{z}\right)} G_{1,2}^{2,1}\left(\frac{\mu_{x} \mu_{z}}{\mu_{y}} \frac{\Omega_{y}}{\Omega_{x} \Omega_{z}} \lambda^{\alpha} \mid \begin{array}{c}
-\frac{1}{2}\left(\mu_{x}+2 \mu_{y}+\mu_{z}-3\right) \\
\frac{1}{2}\left(\mu_{z-} \mu_{x}+1\right), \frac{1}{2}\left(1-\mu_{z+} \mu_{x}\right)
\end{array}\right), \\
F_{\lambda}(\lambda)= & \left(\frac{\mu_{x} \mu_{z}}{\mu_{y}}\right)^{(1 / 2)\left(\mu_{x}+\mu_{z}-1\right)}\left(\frac{\Omega_{y}}{\Omega_{x} \Omega_{z}}\right)^{(1 / 2)\left(\mu_{x}+\mu_{z}-1\right)} \lambda^{(\alpha / 2)\left(\mu_{x}+\mu_{z}-1\right)} \frac{1}{\Gamma\left(\mu_{x}\right) \Gamma\left(\mu_{y}\right) \Gamma\left(\mu_{z}\right)} \\
& \times G_{2,3}^{2,2}\left(\frac{\mu_{x} \mu_{z}}{\mu_{y}} \frac{\Omega_{y}}{\Omega_{x} \Omega_{z}} \lambda^{\alpha} \mid \begin{array}{c}
-\frac{1}{2}\left(\mu_{x}+2 \mu_{y}+\mu_{z}-3\right), \frac{1}{2}\left(3-\mu_{x}-\mu_{z}\right) \\
\frac{1}{2}\left(\mu_{z}-\mu_{x}+1\right), \frac{1}{2}\left(1-\mu_{z}+\mu_{x}\right),-\frac{1}{2}\left(\mu_{x}+\mu_{z}-1\right)
\end{array}\right) .
\end{aligned}
$$

The PDFs and CDFs of $\lambda$ in all considered scenarios are presented in Figures 1, 2, 3 and 4.

\section{An Application of Presented Theoretical Results in Wireless Communication Systems: The Performance Analysis of Multihop Systems}

Multihop (N-hop) transmission is a technique by which the channel from the source (S) to the destination (D) is split into several, possibly shorter, links using relays $\left(R_{i}, i=\overline{1, N-1}\right)$, as shown in Figure 5.

Depending on the nature of the radio propagation environment, the statistical behaviour of signal envelope can be described with different distributions. This is reason 


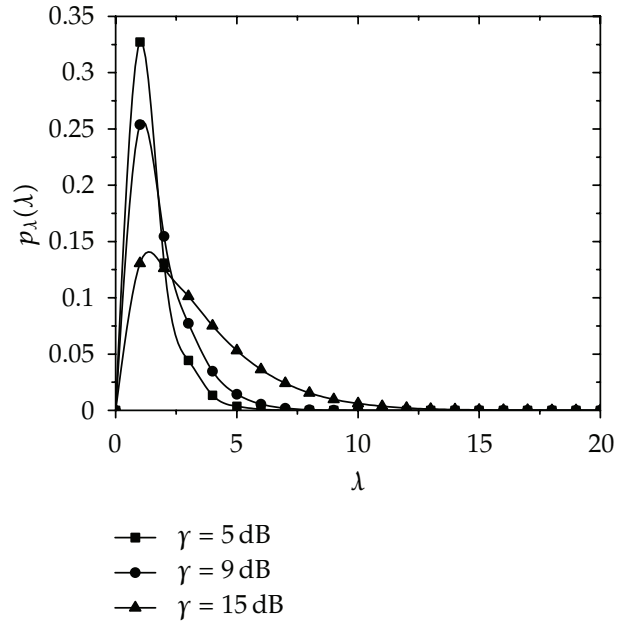

(a)

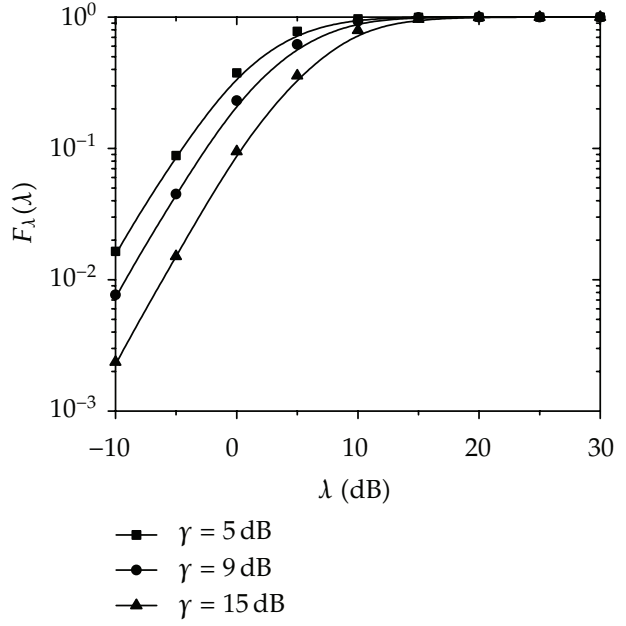

(b)

Figure 1: PDF and CDF of $\lambda$ in Rayleigh scenario.

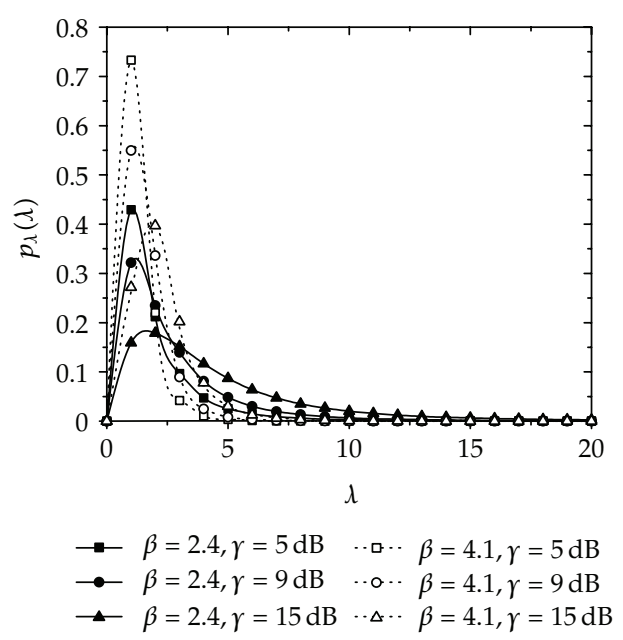

(a)

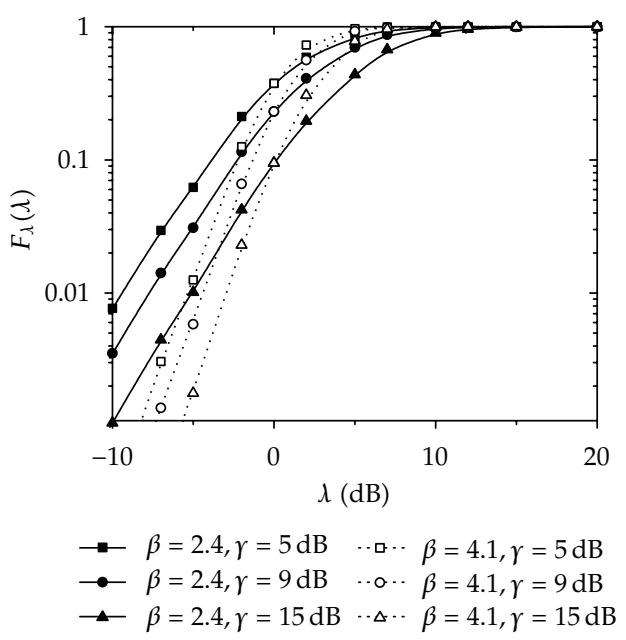

(b)

Figure 2: PDF and CDF of $\lambda$ in Weibull scenario.

why our analysis includes so many statistical models. The Rayleigh model is frequently used to describe multipath fading with no direct line-of-sight (LOS) path [10]. The Weibull distribution exhibits an excellent fit to experimental fading channel measurements, for both indoor [21] and outdoor [22] environments. Also good results are provided in urban environments. The Nakagami- $m$ distribution has gained widespread application in the modelling of physical radio channels since it shows great agreement with experimentally obtained results [23]. Fading severity in Weibull and Nakagami- $m$ environments is described by Weibull parameter $\beta$ and Nakagami parameter $m$, respectively. As these parameters increase fading severity decreases. The $\alpha-\mu$ distribution is a general fading distribution that can be used to better represent the small-scale variation of the fading signal [24]. Parameter 


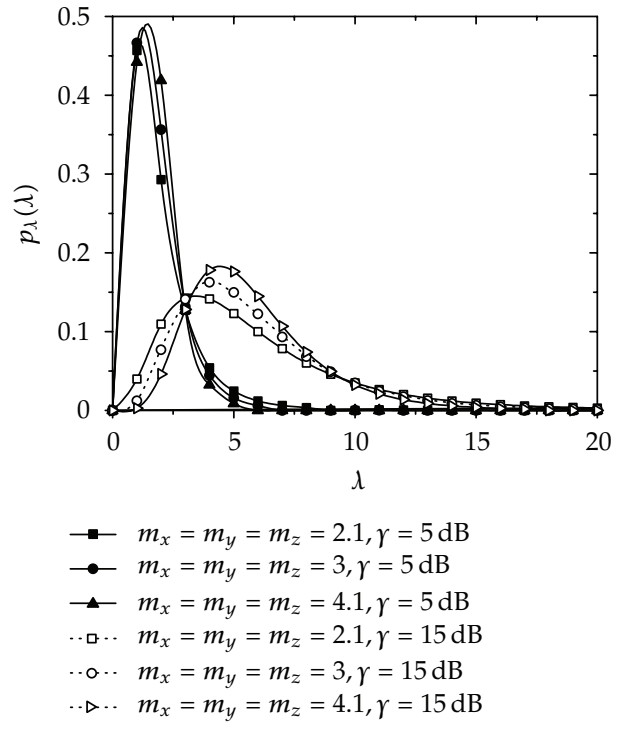

(a)

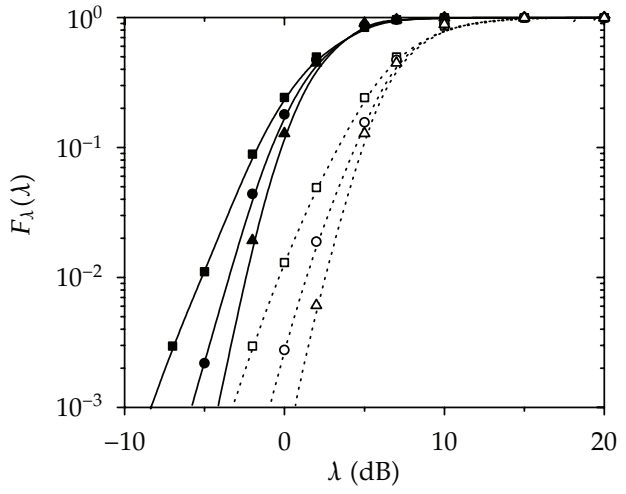

$\longrightarrow m_{x}=m_{y}=m_{z}=2.1, \gamma=5 \mathrm{~dB}$

$\longrightarrow m_{x}=m_{y}=m_{z}=3, \gamma=5 \mathrm{~dB}$

$\neg m_{x}=m_{y}=m_{z}=4.1, \gamma=5 \mathrm{~dB}$

ㅁ. ‥ $m_{x}=m_{y}=m_{z}=2.1, \gamma=15 \mathrm{~dB}$

.०... $m_{x}=m_{y}=m_{z}=3, r=15 \mathrm{~dB}$

$\therefore \Delta . . \quad m_{x}=m_{y}=m_{z}=4.1, \gamma=15 \mathrm{~dB}$

(b)

Figure 3: PDF and CDF of $\lambda$ in Nakagami- $m$ scenario.
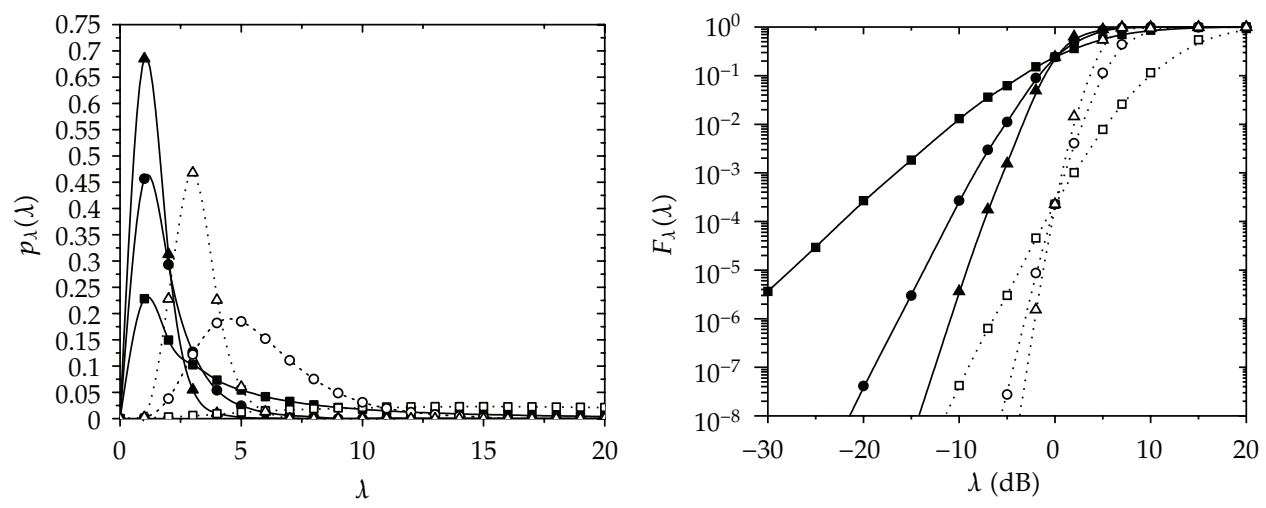

$$
\begin{aligned}
& \rightarrow \alpha=1, \mu_{x}=\mu_{y}=\mu_{z}=2.1, \gamma=5 \mathrm{~dB} \\
& \because \alpha=2, \mu_{x}=\mu_{y}=\mu_{z}=2.1, \gamma=5 \mathrm{~dB} \\
& \rightarrow \alpha=3, \mu_{x}=\mu_{y}=\mu_{z}=2.1, \gamma=5 \mathrm{~dB} \\
& \therefore \text { - } \alpha=1, \mu_{x}=\mu_{y}=\mu_{z}=4.5, \gamma=15 \mathrm{~dB} \\
& \therefore \text {. } \alpha=2, \mu_{x}=\mu_{y}=\mu_{z}=4.5, \gamma=15 \mathrm{~dB} \\
& \therefore \Delta . \quad \alpha=3, \mu_{x}=\mu_{y}=\mu_{z}=4.5, \gamma=15 \mathrm{~dB}
\end{aligned}
$$

(a)

$$
\begin{aligned}
& \rightarrow \alpha=1, \mu_{x}=\mu_{y}=\mu_{z}=2.1, \gamma=5 \mathrm{~dB} \\
& \rightarrow \alpha \alpha=2, \mu_{x}=\mu_{y}=\mu_{z}=2.1, \gamma=5 \mathrm{~dB} \\
& \rightarrow \alpha=3, \mu_{x}=\mu_{y}=\mu_{z}=2.1, \gamma=5 \mathrm{~dB} \\
& \therefore \text {. } \alpha=1, \mu_{x}=\mu_{y}=\mu_{z}=4.5, \gamma=15 \mathrm{~dB} \\
& \therefore \text {. } \alpha=2, \mu_{x}=\mu_{y}=\mu_{z}=4.5, \gamma=15 \mathrm{~dB} \\
& \therefore \Delta . \quad \alpha=3, \mu_{x}=\mu_{y}=\mu_{z}=4.5, \gamma=15 \mathrm{~dB}
\end{aligned}
$$

(b)

Figure 4: PDF and CDF of $\lambda$ in $\alpha-\mu$ scenario.

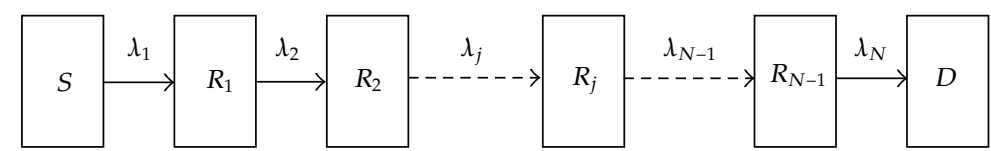

Figure 5: Multihop transmission. 
$\alpha$ is related to the nonlinearity of the environment, while parameter $\mu$ is associated to the number of multipath [25].

The product of random variables, $t_{i}=x_{i} z_{i}$, represents signal envelope which suffers from fading and shadowing while the random variable $y_{i}$ represents CCI envelope at the input of $i$ th terminal $(i=\overline{1, N})$. The random variable $\lambda_{i}=x_{i} z_{i} / y_{i}$ presents SIR value at the input of $i$ th terminal. Therefore, the mathematical results presented in the previous section can be efficiently used for performance analysis of relayed communication systems in fading environment. Highly important and widely accepted system performance indicator is outage probability defined as probability of having SIR value lower than predetermined threshold $\lambda_{0}$ which defines required QoS. The system failure can occur in sections $S-R_{1}, R_{1}$ $R_{2}, R_{2}-R_{3}, \ldots, R_{j-1}-R_{j}, \ldots, R_{N-1}-D$ when some of the values of $\lambda_{1}, \lambda_{2}, \lambda_{3}, \ldots, \lambda_{j}, \ldots, \lambda_{N}$ are below the predetermined threshold $\lambda_{0}$. The PDF of minimum of $\lambda_{i}, \lambda=\min \left\{\lambda_{1}, \lambda_{2}, \ldots, \lambda_{N}\right\}$, is important for analyzing multihop relayed communication systems in which the source terminal communicates with the destination terminal through a number of relay terminals. It can be obtained based on previous results as

$$
p_{\lambda}(\lambda)=\sum_{n=1}^{N} p_{\lambda_{n}}(\lambda) \prod_{\substack{k=1 \\ k \neq n}}^{N}\left(1-F_{\lambda_{k}}(\lambda)\right) .
$$

The outage probability of multihop system is defined as

$$
P_{\text {out }}=\int_{0}^{\lambda_{0}} p_{\lambda}(\lambda) d \lambda
$$

As an illustrative example, the outage probability of dual-hop and triple-hop communication systems can be obtained as

$$
\begin{aligned}
P_{\text {out }}= & F_{\lambda_{1}}(\lambda)\left(1-F_{\lambda_{2}}(\lambda)\right)+F_{\lambda_{2}}(\lambda)\left(1-F_{\lambda_{1}}(\lambda)\right)+F_{\lambda_{1}}(\lambda) F_{\lambda_{2}}(\lambda), \\
P_{\text {out }}= & F_{\lambda_{1}}(\lambda)\left(1-F_{\lambda_{2}}(\lambda)\right)\left(1-F_{\lambda_{3}}(\lambda)\right)+F_{\lambda_{2}}(\lambda)\left(1-F_{\lambda_{1}}(\lambda)\right)\left(1-F_{\lambda_{3}}(\lambda)\right) \\
& +F_{\lambda_{3}}(\lambda)\left(1-F_{\lambda_{1}}(\lambda)\right)\left(1-F_{\lambda_{2}}(\lambda)\right)+F_{\lambda_{1}}(\lambda) F_{\lambda_{2}}(\lambda)\left(1-F_{\lambda_{3}}(\lambda)\right) \\
& +F_{\lambda_{1}}(\lambda) F_{\lambda_{3}}(\lambda)\left(1-F_{\lambda_{2}}(\lambda)\right)+F_{\lambda_{2}}(\lambda) F_{\lambda_{3}}(\lambda)\left(1-F_{\lambda_{1}}(\lambda)\right) \\
& +F_{\lambda_{1}}(\lambda) F_{\lambda_{2}}(\lambda) F_{\lambda_{3}}(\lambda),
\end{aligned}
$$

respectively. The curves representing PDF of $\lambda$ and outage probability of multihop (dualhop and triple-hop) communication system in Weibull fading environment are presented in Figure 6 . Without loss of generality, we assumed that the ratios of average powers are equal on all terminals inputs, that is, $\gamma_{i}=\Omega_{x_{i}} \Omega_{z_{i}} / \Omega_{y_{i}}=\gamma, i=\overline{1, N}$. Namely, signal is amplified in terminal so that the ratio of average powers at the input of the next terminal is equal to the ratio of average powers at the input of previous one. It is noticeable that the outage probability is higher for lower values of Weibull parameter, that is, in environment with higher fading severity. Also, having in mind that $\gamma_{i}=\gamma, i=\overline{1, N}$, the higher values of $N$ imply larger distance between the source and destination terminals and higher values of the outage probability. 


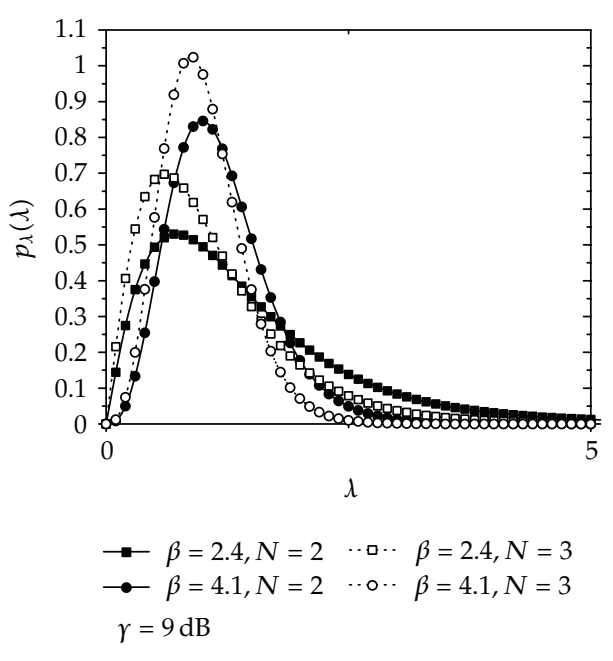

(a)

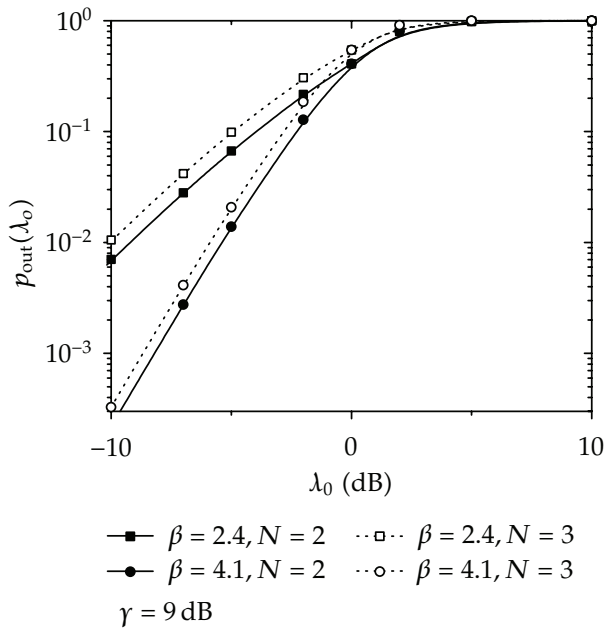

(b)

Figure 6: PDF of $\lambda=\min \left\{\lambda_{1}, \lambda_{2}, \ldots, \lambda_{N}\right\}$ and outage probability of dual-hop $(N=2)$ and triple-hop $(N=3)$ communication system for different values of $\gamma$ in Weibull fading environment.

\section{Conclusion}

The PDF and CDF of ratio of product of two random variables and random variable $\lambda=$ $x z / y=t / y$ have been derived. Rayleigh, Weibull, Nakagami- $m$, and $\alpha-\mu$ statistical models are included in the paper, so that other researchers and engineers could use our results in wide range of scenarios in many areas of science. An application of these results for the wireless communications community has been described. Namely, presented results can help the designers of wireless communication systems to simulate different wireless environments where fading and shadowing affect desired signal and fading affects CCI and readjust system parameters in order to meet the QoS demands. In our future work, we hope to obtain analytical expressions for PDF and CDF of ratio of products of random variables which will have application in analysis of wireless communication systems where both desired signal and CCI are affected by fading and shadowing simultaneously which is realistic scenario in modern urban areas.

\section{Acknowledgment}

This work was supported by the Ministry of Science of Serbia within the Project TR-32023.

\section{References}

[1] S. Nadarajah and S. Kotz, "On the ratio of Pearson type VII and Bessel random variables," Journal of Applied Mathematics and Decision Sciences, vol. 2005, no. 4, pp. 191-199, 2005.

[2] A.P. Basu and R. H. Lochner, "On the distribution of the ratio of two random variables having generalized life distributions," Technometrics, vol. 13, pp. 281-287, 1971.

[3] T. Pham-Gia, "Distributions of the ratios of independent beta variables and applications," Communications in Statistics - Theory and Methods, vol. 29, no. 12, pp. 2693-2715, 2000.

[4] P. J. Korhonen and S. C. Narula, "The probability distribution of the ratio of the absolute values of two normal variables," Journal of Statistical Computation and Simulation, vol. 33, pp. 173-182, 1989. 
[5] G. Marsaglia, "Ratios of normal variables and ratios of sums of uniform variables," Journal of the American Statistical Association, vol. 60, pp. 193-204, 1965.

[6] S. Okui, "Effects of CIR selection diversity with two correlated branches in the m-fading channel," IEEE Transactions on Communications, vol. 48, no. 10, pp. 1631-1633, 2000.

[7] M. C. Stefanovič, D. L. Draća, A. S. Panajotovič, and N. M. Sekulovič, "Performance analysis of system with L-branch selection combining over correlated Weibull fading channels in the presence of cochannel interference," International Journal of Communication Systems, vol. 23, no. 2, pp. 139-150, 2010.

[8] G. K. Karagiannidis, "Performance analysis of SIR-based dual selection diversity over correlated Nakagami-m fading channels," IEEE Transactions on Vehicular Technology, vol. 52, no. 5, pp. 1207-1216, 2003.

[9] A. S. Panajotović, M. Č. Stefanović, and D. L. Drača, "Performance analysis of system with selection combining over correlated Rician fading channels in the presence of cochannel interference," International Journal of Electronics and Communications, vol. 63, no. 12, pp. 1061-1066, 2009.

[10] M. K. Simon and M.-S. Alouini, Digital Communication over Fading Channels, Wiley, New York, NY, USA, 1st edition, 2000.

[11] G. E. Corazza and F. Vatalaro, "Statistical model for land mobile satellite channels and its application to nongeostationary orbit systems," IEEE Transactions on Vehicular Technology, vol. 43, no. 3, pp. 738742, 1994.

[12] P. G. Babalis and C. N. Capsalis, "Impact of the combined slow and fast fading channel characteristics on the symbol error probability for multipath dispersionless channel characterized by a small number of dominant paths," IEEE Transactions on Communications, vol. 47, no. 5, pp. 653-657, 1999.

[13] J. D. Parsons, The Mobile Radio Propagation Channels, Wiley, New York, NY, USA, 2nd edition, 2000.

[14] G. K. Karagiannidis, T. A. Tsiftsis, and R. K. Mallik, “Bounds for multihop relayed communications in Nakagami-m fading," IEEE Transactions on Communications, vol. 54, no. 1, pp. 18-22, 2006.

[15] M. O. Hasna and M. S. Alouini, "End-to-end performance of transmission systems with relays over Rayleigh-fading channels," IEEE Transactions on Wireless Communications, vol. 2, no. 6, pp. 1126-1131, 2003.

[16] M. O. Hasna and M. S. Alouini, “Outage probability of multihop transmission over Nakagami fading channels," IEEE Communications Letters, vol. 7, no. 5, pp. 216-218, 2003.

[17] C. Zhong, S. Jin, and K. K. Wong, "Outage probability of dual-hop relay channels in the presence of interference," in 69th IEEE Vehicular Technology Conference (VTC '09), pp. 1-5, April 2009.

[18] I. S. Gradshteyn and I. M. Ryzhik, Table of Integrals, Series, and Products, Academic, New York, NY, USA, 5th edition, 1994.

[19] http:/ / functions.wolfram.com/07.45.26.0005.01.

[20] V. S. Adamchik and O. I. Marichev, "Algorithm for calculating integrals of hypergeometric type functions and its realization in reduce system," in Proceedings of International Symposium on Symbolic and Algebraic Computation (ISSAC'90), pp. 212-224, August 1990.

[21] F. Babich and G. Lombardi, "Statistical analysis and characterization of the indoor propagation channel," IEEE Transactions on Communications, vol. 48, no. 3, pp. 455-464, 2000.

[22] G. Tzeremes and C. G. Christodoulou, "Use of Weibull distribution for describing outdoor multipath fading," in IEEE Antennas and Propagation Society International Symposium, pp. 232-235, June 2002.

[23] M. Nakagami, "The $m$-distribution - a general formula of intensity distribution of rapid fading," in Statistical Methods in Radio Wave Propagation, pp. 3-36, Pergamon Press, Oxford, UK, 1960.

[24] M. D. Yacoub, "The $\alpha-\mu$ distribution: a general fading distribution," in 13th IEEE International Symposium on Personal, Indoor and Mobile Radio Communications (PIMRC '02), vol. 2, pp. 629-633, September 2002.

[25] D. B. Da Costa, M. D. Yacoub, and G. Fraidenraich, "Second-order statistics of equal-gain and maximal-ratio combining for the $\alpha-\mu$ (generalized gamma) fading distribution," in 9th IEEE International Symposium on Spread Spectrum Symposium on Spread Spectrum Techniques and Applications (ISSSTA '06), pp. 342-346, August 2006. 


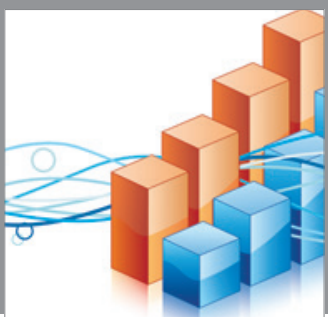

Advances in

Operations Research

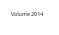

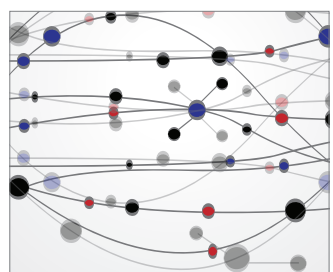

\section{The Scientific} World Journal
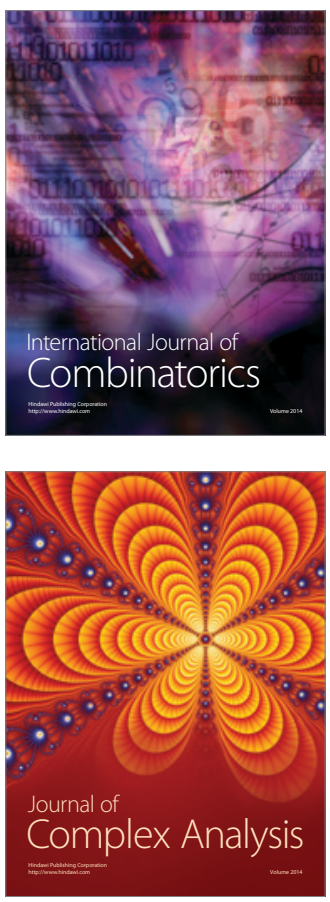

International Journal of

Mathematics and

Mathematical

Sciences
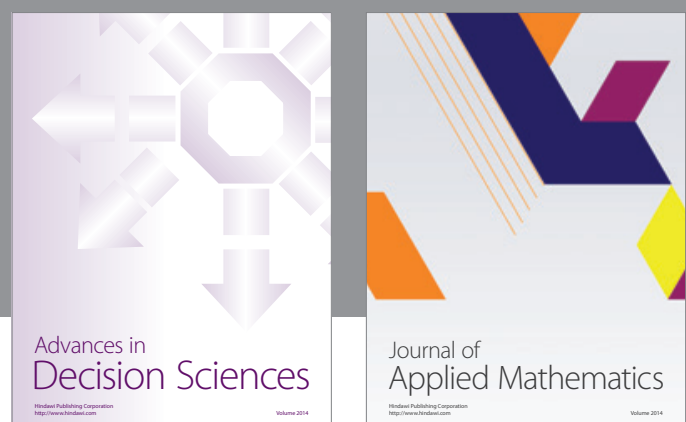

Journal of

Applied Mathematics
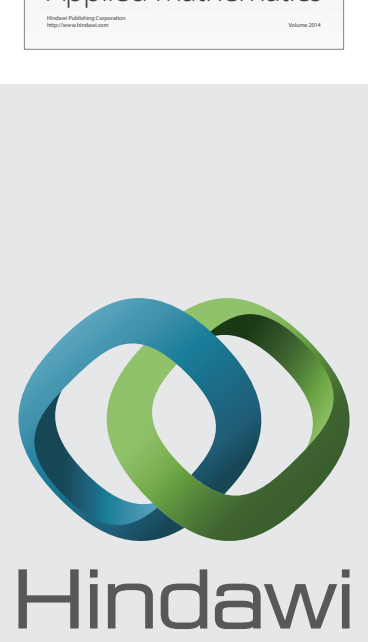

Submit your manuscripts at http://www.hindawi.com
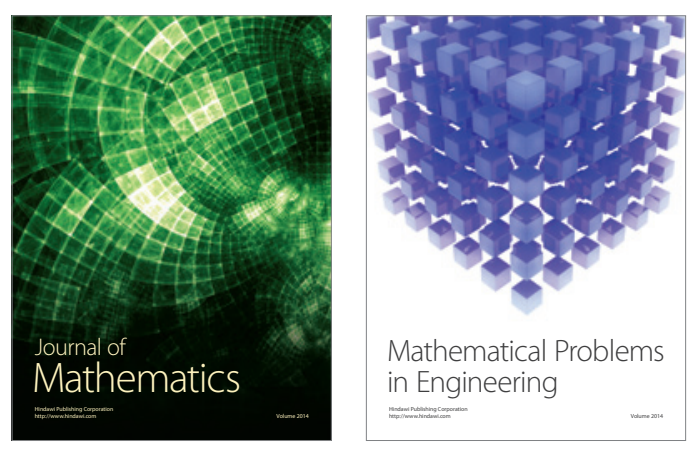

Mathematical Problems in Engineering
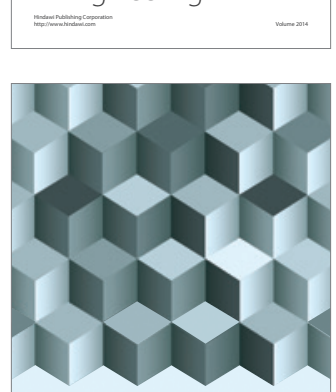

Journal of

Function Spaces
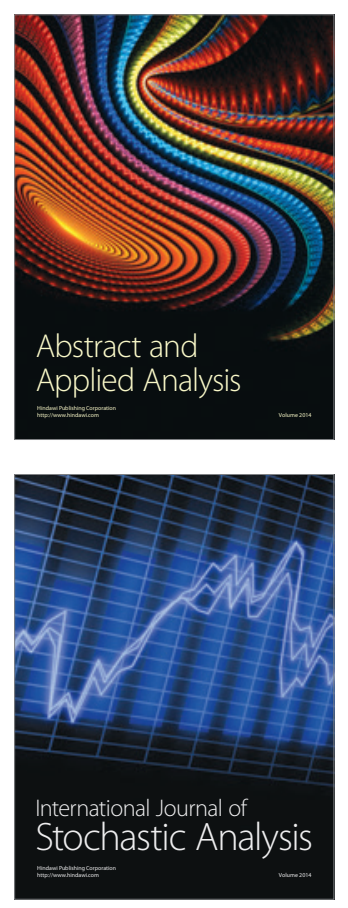

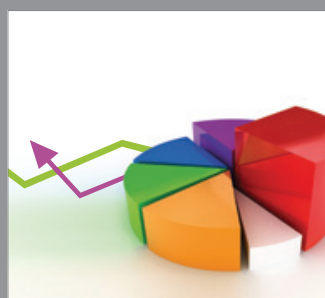

ournal of

Probability and Statistics

Promensencen
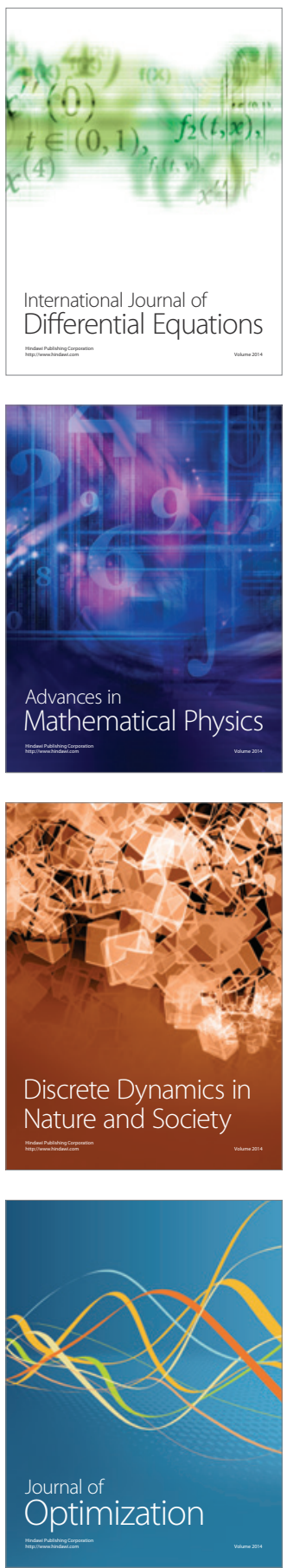